\title{
GEOMETRY OF BOUNDED DOMAINS
}

\author{
BY
}

\section{SHOSHICHI KOBAYASHI}

In this paper, we shall study differential geometric properties of bounded domains in $C^{n}$. Here is the summary of our results.

We consider an $n$-dimensional complex manifold $M$ and the Hilbert space of square integrable holomorphic $n$-forms on $M$. After Bergman $[3 ; 4 ; 5]$, we define the kernel form on $M$ (instead of the kernel function) and, under certain assumptions, we define the invariant metric of Bergman. This method of generalizing the theory of S. Bergman (although the generalization is not essential) allows us to define the Bergman metric on certain compact complex manifolds.

Some elementary properties of the kernel form and the Bergman metric (mostly already classical) are studied for the sake of completeness. Then we reexamine the theorem of $\mathrm{H}$. Cartan on the group of holomorphic transformations of a bounded domain from the differential geometric point of view.

We study also differential geometric properties of a manifold $M$ which admits a discontinuous group $D$ of holomorphic transformations such that $M / D$ is compact. It should be noted that such a manifold possesses properties similar to those of a homogeneous manifold.

Bremermann has studied the bounded domains with the following property $(\mathrm{P})$ : the kernel function goes to infinity at every boundary point [8]. He has shown that a bounded domain with the property $(\mathrm{P})$ is a domain of holomorphy and that the converse is not true. (The same result has been obtained also by Sommer and Mehring [22].) Making use of this result, he has proved that if a bounded domain is complete with respect to the Bergman metric, then it is a domain of holomorphy. Since the kernel function is not intrinsically defined, the property $(\mathrm{P})$ is not intrinsic. We consider, therefore, a condition which is stronger than $(\mathrm{P})$ but which is intrinsic. This condition can be roughly stated as follows: if $K$ is the kernel form and $f$ is a square integrable holomorphic $n$-form, then $(f \wedge \bar{f}) / K$ goes to zero at every boundary point. We shall prove that this condition implies the completeness of the Bergman metric. From this result it can be proved, for instance, that every bounded analytic polyhedron is complete with respect to the Bergman metric.

As a preparation for the above study of "completeness," we shall prove that a manifold with the Bergman metric can be isometrically imbedded, in a natural way, into a complex projective space (of infinite dimension, in general).

Finally, we shall give examples of manifolds (beside the classical bounded

Received by the editors February 6, 1958. 
domains) which admit the Bergman metric.

Before we finish this introduction, we add some remarks. The idea of using the square integrable holomorphic $n$-forms can be found in [28] and has been discussed by E. Calabi at the Summer Institute on Differential Geometry held in Seattle in 1956. An example of algebraic manifold admitting the Bergman metric is due to Calabi.

1. Kernel functions and invariant metrics of Bergman. In this section we recall the definition of the kernel function and the invariant metric of a bounded domain in the complex $n$-space $C^{n}$ due to S. Bergman.

Let $M$ be a bounded domain (i.e., a connected open set) in $C^{n}$ with coordinate system $z^{1}, \cdots, z^{n}$. The set $F$ of all holomorphic functions $f$ on $M$ which are square integrable forms a separable complex Hilbert space. Let $h_{0}, h_{1}, h_{2}, \cdots$ be an orthonormal basis for $F$. Then

$$
K(z, \bar{w})=\sum_{j=0}^{\infty} h_{j}(z) \bar{h}_{j}(\bar{w})
$$

is a holomorphic function in $z$ and $\bar{w}$, or a holomorphic function on $M \times \bar{M}$, where $\bar{M}$ is the manifold conjugate to $M$, and is called the kernel function of Bergman. Define

$$
d s^{2}=\sum \frac{\partial^{2} \log K(z, z)}{\partial z^{\alpha} \partial \bar{z}^{\beta}} d z^{\alpha} d \bar{z}^{\beta} .
$$

Then $d s^{2}$ is a positive definite Kaehler metric on $M$ which is invariant under the holomorphic transformations of $M$. This metric, which is associated intrinsically with the complex structure of the manifold $M$, is called the Bergman metric.

Note that, in the above definition of $F$, we made use of a coordinate system $z^{1}, \cdots, z^{n}$ which is well defined throughout $M$. Given a complex manifold $M$ (not necessarily a bounded domain), we do not have, in general, a coordinate system in the large. We consider, therefore, holomorphic $n$-forms instead of holomorphic functions.

2. Kernel forms. Let $M$ be a complex $n$-dimensional manifold. Let $F$ be the set of holomorphic $n$-forms $f$ on $M$ such that

$$
\left|\int_{M} f \wedge \bar{f}\right|<\infty \text {. }
$$

The vector space $F$ is a separable complex Hilbert space with an inner product given by

$$
\left(f_{1}, f_{2}\right)=(-1)^{n^{2} / 2} \int_{M} f_{1} \wedge \bar{f}_{2}
$$

It should be noted that $F$ may be finite dimensional (this is the case if $M$ is compact). 
Let $h_{0}, h_{1}, h_{2}, \cdots$ be a complete orthonormal basis for $F$. Then

$$
K(z, \bar{w})=\sum_{j=0}^{\infty} h_{j}(z) \wedge \bar{h}_{j}(\bar{w})
$$

is a holomorphic $2 n$-form on $M \times \bar{M}$, where $\bar{M}$ is the complex manifold conjugate to $M$, and it is independent of choice of orthonormal basis. We call $K$ the kernel form of $M$.

If $\bar{z}$ is the point of $\bar{M}$ corresponding to a point $z$ of $M$, the set of points $(z, \bar{z})$ of $M \times \bar{M}$ is identified, in a natural fashion, with $M$. Hence, $K(z, \bar{z})$ can be considered as a $2 n$-form on $M$.

TheOREM 2.1. The form $K(z, \bar{z})$ is invariant under the group of holomorphic transformations of $M$.

Proof. Let $\phi$ be any holomorphic transformation of $M$. If $h_{0}, h_{1}, h_{2}, \cdots$ forms a complete orthonormal basis for $F$, so does $\phi^{*}\left(h_{0}\right), \phi^{*}\left(h_{1}\right), \phi^{*}\left(h_{2}\right), \cdots$, as it can be easily verified. Since the kernel form is independent of choice of orthonormal basis for $F$, we have

$$
K(z, \bar{z})=\sum \phi^{*}\left(h_{j}(z)\right) \wedge \phi^{*}\left(\bar{h}_{j}(\bar{z})\right)=\phi^{*}(K(z, \bar{z})) .
$$

Let $f_{1}, f_{2} \in F$. Then, for every point $z$ of $M$, there exist real numbers $\left(c_{1}, c_{2}\right) \neq(0,0)$ such that

$$
c_{1} f_{1} \wedge \bar{f}_{1}=c_{2} f_{2} \wedge \bar{f}_{2}
$$

If $c_{1}=0$ or $c_{2} / c_{1} \geqq 1$, then we say that $f_{1} \wedge \bar{f}_{1} \geqq f_{2} \wedge \bar{f}_{2}$ at $z$.

THEOREM 2.2 .

$$
K(z, \bar{z})=\operatorname{Max}_{(f, f)=1} f(z) \wedge \bar{f}(\bar{z}) .
$$

If $K(z, \bar{z}) \neq 0$, then an $n$-form $f \in F$ satisfying the above equality is unique up to a constant factor $c$ with $|c|=1$ and is characterized by the following two properties:

(a) $(f, f)=1$.

(b) $\left(f, f^{\prime}\right)=0$ for all $f^{\prime} \in F$ which vanish at $z$.

Proof. Fix a point $z$ in $M$ and let $F^{\prime}$ be the set of $n$-forms $f^{\prime} \in F$ which vanish at $z$. If $F^{\prime}=F$, then our theorem is trivial. Suppose $F^{\prime} \neq F$. Let $h_{0}$ be an element of $F$ which is orthogonal to $F^{\prime}$ and has the unit length. Let $g$ be any element of $F$ such that $g(z) \neq 0$. Let $c$ be a complex number satisfying $g(z)=c h_{0}(z)$. Then $g-c h_{0}$ is in $F^{\prime}$. Hence, $F$ is spanned by $F^{\prime}$ and $h_{0}$. Given $z$ in $M$, we can choose, therefore a complete orthonormal basis $h_{0}, h_{1}, h_{2}, \ldots$ for $F$ in such a way that $h_{0}(z) \neq 0, h_{1}(z)=h_{2}(z)=\cdots=0$. This implies immediately Theorem 2.2 .

From Theorem 2.2 we obtain the following 
TheOREM 2.3. Let $M^{\prime}$ be a domain (connected open subset) in $M$ and let $K_{M}$ and $K_{M^{\prime}}$ be the kernel forms of $M$ and $M^{\prime}$ respectively. Then

$$
K_{M} \leqq K_{M^{\prime}}
$$

on $M^{\prime}$

in the sense that there exists a function $c$ on $M^{\prime}$ such that $K_{M}=c K_{M^{\prime}}$ and $0 \leqq c \leqq 1$ on $M^{\prime}$.

REMARK. If $M-M^{\prime}$ contains an open set (nonempty) in $M$, then either $K_{M^{\prime}}(z, \bar{z})>K_{M}(z, \bar{z})$ or $K_{M^{\prime}}(z, \bar{z})=K_{M}(z, \bar{z})=0$ for each $z$ of $M^{\prime}$.

On the other hand, we have the following

Theorem 2.4. If $M^{\prime}$ is a domain in $M$ and if $M-M^{\prime}$ is an analytic subvariety of $M$ of complex dimension $\leqq n-1$, then

$$
K_{M^{\prime}}(z, \bar{z})=K_{M}(z, \bar{z}) \quad \text { for all } z \text { in } M^{\prime} .
$$

Proof. Let $f$ be a square integrable holomorphic $n$-form on $M^{\prime}$. We shall show that $f$ can be extended holomorphically to $M$. Let $z_{0}$ be any nonsingular point of a variety $M-M^{\prime}$. Let $z^{1}, \cdots, z^{n}$ be a local coordinate system in a neighborhood $U$ of $z_{0}$ in $M$ such that $\left(M-M^{\prime}\right) \cap U$ is given by $z^{1}=\cdots=z^{k}$ $=0$ and $z_{0}$ is the origin of the coordinate system. Let $f$ be written in $M^{\prime} \cap U$ as follows:

$$
f=f^{*} d z^{1} \wedge \cdots \wedge d z^{n},
$$

where $f^{*}$ is a function holomorphic in $M^{\prime} \cap U$. Let $V$ be the plane defined by

$$
z^{2}=z^{3}=\cdots=z^{n}=0 .
$$

Then $f^{*}$ is a function holomorphic on $V-\left\{z_{0}\right\}$ and can be expanded in a Laurent series in $z^{1}$ around the origin. Since $f$ is square integrable,

$$
\int_{V-\left\{z_{0}\right\}} f^{*} d z^{1} \wedge d \bar{z}^{1}
$$

has to be finite. (It is, of course, essential here that $f^{*}$ is holomorphic.) From this, we can conclude that $f^{*}$ is a power series in $z^{1}$ around the origin. (See the reasoning of p. 363 in [8].) Hence $f$ can be continued holomorphically to $M^{\prime \prime}$, where $M^{\prime \prime}$ is the union of $M^{\prime}$ and the nonsingular points of $\left(M-M^{\prime}\right)$. Since $M-M^{\prime \prime}$ is a subvariety of $M$ and $\operatorname{dim}\left(M-M^{\prime \prime}\right)<\operatorname{dim}\left(M-M^{\prime}\right)$, we can conclude our theorem by induction.

Theorem 2.5. Let $M$ and $M^{\prime}$ be complex manifolds of complex dimension $n$ and $n^{\prime}$ respectively. Then

$$
K_{M \times M^{\prime}}=(-1)^{n n^{\prime}} K_{M} \wedge K_{M^{\prime}} .
$$

Proof. In the above formula, the projections from $M \times M^{\prime}$ onto $M$ and $M^{\prime}$ are omitted; $K_{M}$ and $K_{M^{\prime}}$ can be considered as forms on $M \times M^{\prime}$ in a natural manner. 
Let $z_{0} \in M$ and $z_{0}^{\prime} \in M^{\prime}$. From Theorem 2.2, it follows that there exist forms $h, h^{\prime}$ and $h^{\prime \prime}$ on $M, M^{\prime}$ and $M \times M^{\prime}$ respectively such that

$$
\begin{array}{rlrl}
(h, h) & =\left(h^{\prime}, h^{\prime}\right)=\left(h^{\prime \prime}, h^{\prime \prime}\right)=1, \\
K_{M} & =h \wedge \bar{h} & & \text { at } z_{0}, \\
K_{M^{\prime}} & =h^{\prime} \wedge \bar{h}^{\prime} & & \text { at } z_{0}^{\prime}, \\
K_{M \times M^{\prime}} & =h^{\prime \prime} \wedge \bar{h}^{\prime \prime} & & \text { at }\left(z_{0}, z_{0}^{\prime}\right) .
\end{array}
$$

If $K_{M \times M^{\prime}}$ vanishes at $\left(z_{0}, z_{0}^{\prime}\right)$, then our theorem is trivial. If it does not vanish, then $h^{\prime \prime}$ is characterized by the properties:

$\left(h^{\prime \prime}, h^{\prime \prime}\right)=1$ and $\left(h^{\prime \prime}, f^{\prime \prime}\right)=0$ for every $f^{\prime \prime}$ vanishing at $\left(z_{0}, z_{0}^{\prime}\right)$. More precisely, $h^{\prime \prime}$ defined by the above two properties is unique up to a constant factor $c$ with $|c|=1$; however, $h^{\prime \prime} \wedge \bar{h}^{\prime \prime}$ is unique. It is easy to see that the form $(-1)^{n n^{\prime}} \wedge h^{\prime}$ on $M \times M^{\prime}$ possesses the above two properties.

Remark. Compare the proofs of Bremermann [8] and Morita [24].

3. Invariant metric. Let $M$ be a complex manifold of complex dimension $\boldsymbol{n}$. Suppose that

(A.1) Given any point $z$ of $M$, there exists a square integrable holomorphic $n$-form $f$ such that $f(z) \neq 0$. In other words, the kernel form $K(z, \bar{z})$ of $M$ is different from zero at every point of $M$.

Let $z^{1}, \cdots, z^{n}$ be a local coordinate system in $M$. Let

$$
K(z, \bar{z})=K^{*}(z, \bar{z}) d z^{1} \wedge \cdots \wedge d z^{n} \wedge d \bar{z}^{1} \wedge \cdots \wedge d \bar{z}^{n},
$$

where $K^{*}(z, \bar{z})$ is a locally defined function. Define a quadratic (hermitian) differential form $d s^{2}$ by

$$
d s^{2}=\sum \frac{\partial^{2} \log K^{*}}{\partial z^{\alpha} \partial \bar{z}^{\beta}} d z^{\alpha} d \bar{z}^{\beta} .
$$

It can easily be shown that $d s^{2}$ is independent of choice of coordinate system.

THEOREM 3.1. The quadratic form $d s^{2}$ is positive semidefinite and invariant under the holomorphic transformations of $M$.

Proof. Let $z$ be any point of $M$ and let $z^{1}, \cdots, z^{n}$ be a local coordinate system around $z$. Let

$$
h_{j}=h_{j}^{*} d z^{1} \wedge \cdots \wedge d z^{n}, \quad j=0,1,2, \cdots,
$$

be an orthonormal basis for $F$ such that

$$
h_{0}(z) \neq 0, \quad h_{1}(z)=h_{2}(z)=\cdots=0 .
$$

(See the proof of Theorem 2.2.) Then, from $K^{*}=\sum h_{j}^{*} \bar{h}_{j}^{*}$, it follows, by a simple calculation, that

$$
d s^{2}=\left(\sum_{k=1}^{\infty} d h_{k}^{*} \cdot d \bar{h}_{k}^{*}\right) / K^{*} \quad \text { at } z .
$$


This shows that $d s^{2}$ is positive semidefinite.

In order to prove that $d s^{2}$ is invariant by a holomorphic transformation $\phi$ of $M$, let $w^{1}, \cdots, w^{n}$ be a coordinate system around $\phi(z)$ :

$$
\phi: w^{j}=\phi^{i}\left(z^{1}, \cdots, z^{n}\right) \quad j=1, \cdots, n .
$$

Let

$$
K=K^{*} d z^{1} \wedge \cdots \wedge d \bar{z}^{n}=L^{*} d w^{1} \wedge \cdots \wedge d \bar{w}^{n} .
$$

From Theorem 2.1, we obtain

$$
K^{*}(z, \bar{z})=L^{*}(\phi(z), \bar{\phi}(\bar{z})) \cdot J \bar{J},
$$

where $J$ is the Jacobian $\partial \phi^{i} / \partial z^{k}$. Now, the invariance of $d s^{2}$ follows from the definition of $d s^{2}$ and the analyticity of $J$.

From the proof of Theorem 3.1, we can see easily that $d s^{2}$ is positive definite if and only if the following assumption is satisfied:

(A.2) For every holomorphic vector $Z$ at $z$, there exists a square integrable holomorphic $n$-form $f$ such that $f(z)=0$ and $Z\left(f^{*}\right) \neq 0$, where $f=f^{*} d z^{1} \wedge \cdots$ $\wedge d z^{n}$.

By a holomorphic vector $Z$ at $z$, we mean a complex tangent vector of the form:

$$
Z=\sum \zeta^{j}\left(\partial / \partial z^{i}\right)_{z},
$$

where the $\zeta^{i}$ 's are complex numbers.

The metric $d s^{2}$ thus obtained is Kaehlerian and is called the invariant metric of Bergman (or the Bergman metric) of $M$.

As an immediate consequence of Theorem 2.5, we have the

TheOREM 3.2. Let $M$ and $M^{\prime}$ be complex manifolds satisfying (A.1 and 2) and let $d s^{2}$ and $d s^{\prime 2}$ be the Bergman metrics of $M$ and $M^{\prime}$ respectively. Then the Bergman metric of $M \times M^{\prime}$ is $d s^{2}+d s^{\prime 2}$.

From Theorem 2.4, we obtain the following

Theorem 3.3. Let $M^{\prime}$ be a domain in $M$ such that $M-M^{\prime}$ is an analytic subvariety of $M$ of complex dimension $\leqq n-1$ (where $n=\operatorname{dim} M$ ). Then the Bergman metric of $M^{\prime}$ is the restriction to $M^{\prime}$ of the Bergman metric of $M$.

Let $G(M)$ be the group of holomorphic transformations of $M$ with (A. 1 and 2). Then $G(M)$ is a closed subgroup of the group of isometries of $M$ with the Bergman metric. Since the group of isometries of a Riemannian space is a Lie group with compact isotropy group at every point, we obtain the following

Theorem 3.4. Let $M$ be a complex manifold with (A.1 and 2). Then the group $G(M)$ of holomorphic transformations of $M$ is a Lie group. Moreover, the isotropy subgroup of $G(M)$ at every point of $M$ is compact. 
Remark. This generalizes the theorem of H. Cartan for bounded domains. It has been proved by Meyers and Steenrod [23] that the group of isometries of a Riemannian space is a Lie group and the isotropy group at every point of $M$ is compact. Their proof can be nowadays simplified by the theory of connections. The Riemannian connection gives rise to an absolute parallelism on the space of orthonormal frames of $M$. The group of isometries of $M$ can be identified with the group of transformations leaving the absolute parallelism of the space $P$ of orthonormal frames of $M$. By this method, we can imbed the group as a closed submanifold of $P$ in a natural way, thus establishing the theorem of Meyers and Steenrod. The details can be found in $[15 ; 16]$.

We shall show that the differential geometric method is effective also for the proof of the following theorem of H. Cartan[10].

Theorem 3.5. Let $M$ be a bounded domain in the complex $n$-space $C^{n}$. Let $X$ be a nonzero holomorphic vector field on $M$ which generates a 1-parameter group of global holomorphic transformations of $M$ (i.e., $X$ generates a 1-parameter subgroup of $G(M)$ ). Then the holomorphic vector field $i X$ on $M$ does not generate global transformations of $M$.

In order to prove the above theorem, we consider a general case: let $M$ be a complex manifold with (A.1 and 2). Assume holomorphic vector fields $X$ and $i X$ on $M$ both generate 1-parameter subgroups of $G(M)$. Since the Bergman metric is invariant by $G(M)$, both $X$ and $i X$ are infinitesimal isometries (Killing vector fields) of $M$.

On the other hand we have the following general

TheOREM 3.6. Holomorphic vector fields $X$ and $i X$ are both Killing vector fields if and only if they are parallel vector fields. (This Theorem holds for any Kaehlerian manifold.)

Proof is a simple calculation, hence omitted.

The integral submanifold defined by $X$ and $i X$ is a complex 1 -dimensional flat Kaehlerian manifold. It is, moreover, complete in the sense of differential geometry, because $X$ and $i X$ generate 1-parameter group of global transformations. (Here, it is essential that the submanifold is flat.) Therefore, the universal covering space of the submanifold is $C$.

Theorem 3.7. Let $M$ be a complex manifold with (A.1 and 2). Assume that one of the following conditions is satisfied. $M$.

(a). There is no parallel vector field (with respect to the Bergman metric) on

(b). There is no holomorphic map of $C$ into $M$ except constant maps.

(c). There is a point of $M$ where the Ricci curvature is a nonsingular Hermitian form. 
Then, it is impossible that nonzero holomorphic vector fields $X$ and $i X$ on $M$ generate 1-parameter subgroups of $G(M)$.

Proof. We have shown that (a) or (b) implies the theorem. It is also obvious that (c) implies (a).

REMARK. If $M$ is bounded domain in $C^{n}$, then (b) is satisfied. Hence, Theorem 3.5 is an immediate consequence of Theorem 3.7. I do not know whether Theorem 3.7 holds without conditions (a), (b) or (c). It is not known whether (c) is always satisfied or not, even for the classical bounded domains. It will be shown later that (c) is satisfied in some important cases.

4. Ricci curvature and Riemannian curvature. Let $M$ be a complex manifold with (A.1 and 2) and let $d s^{2}=\sum g_{\alpha \bar{\beta}} d z^{\alpha} d \bar{z}^{\beta}$ be the Bergman metric. Let $g=\operatorname{det}\left(g_{\alpha \vec{\beta}}\right)$. Then

$$
v=(-1)^{n^{2} / 2} g d z^{1} \wedge \cdots \wedge d z^{n} \wedge d \bar{z}^{1} \wedge \cdots \wedge d \bar{z}^{n}
$$

is the volume element of the Kaehlerian manifold $M$. The Ricci curvature $R_{\alpha \bar{\beta}}$ is given by

$$
R_{\alpha \bar{\beta}}=-\frac{\partial^{2} \log g}{\partial z^{\alpha} \partial \bar{z}^{\beta}} .
$$

If the group $G(M)$ of holomorphic transformations is transitive on $M$, then

$$
K=c v,
$$

where $c$ is a constant; because both $K$ and $v$ are invariant by $G(M)$. Hence

$$
g_{\alpha \bar{\beta}}=-R_{\alpha \bar{\beta}} .
$$

Thus, we obtain the following well known

Theorem 4.1. Let $M$ be a complex manifold with (A.1 and 2). If the group of holomorphic transformations is transitive on $M$, then

$$
g_{\alpha \bar{\beta}}=-R_{\alpha \bar{\beta}} .
$$

The following theorem is also well known $[17 ; 21 ; 26]$.

THEOREM 4.2. Let $M$ be an arbitrary Kaehlerian manifold. If the Ricci curvature of $M$ is nonsingular (i.e., $\left.\operatorname{det}\left(R_{\alpha \bar{\beta}}\right) \neq 0\right)$ at some point of $M$, then the largest connected group of isometries of $M$ is contained in the group of holomorphic transformations of $M$.

Combining Theorem 4.1 and Theorem 4.2, we obtain

TheOREM 4.3. Let $M$ be a complex manifold with (A.1 and 2) on which the group of holomorphic transformations is transitive. Then the largest connected group of isometries of $M$ is the same as the largest connected group of holomorphic transformations of $M$. 
In order to calculate the Riemannian curvature of a complex manifold $M$ with (A.1 and 2), we set

$$
L=\log K^{*} \quad\left(\text { where } K=K^{*} d z^{1} \wedge \cdots \wedge d z^{n} \wedge d \bar{z}^{1} \wedge \cdots \wedge d \bar{z}^{n}\right) .
$$

We use the following notations:

$$
L_{\alpha}=\partial L / \partial z^{\alpha}, \quad L_{\bar{\beta}}=\partial L / \partial \bar{z}^{\beta}, \quad L_{\alpha \bar{\beta}}=\partial^{2} L / \partial z^{\alpha} \partial \bar{z}^{\beta}, \quad K_{\alpha}^{*}=\partial K^{*} / \partial z^{\alpha} \text {, etc. }
$$

Then the metric tensor and the Riemannian curvature tensor are given by

$$
\begin{aligned}
g_{\alpha \bar{\beta}} & =L_{\alpha \bar{\beta}}, \\
R_{\alpha \bar{\beta} \bar{\gamma} \bar{\delta}} & =-L_{\alpha \bar{\beta} \bar{\gamma} \bar{\delta}}+\sum g^{\bar{\epsilon} \tau} L_{\alpha \gamma \bar{\epsilon}} L_{\bar{\beta} \bar{\delta} \tau} .
\end{aligned}
$$

A straightforward (but long) calculation shows that

$$
\begin{aligned}
R_{\alpha \bar{\beta} \gamma \bar{\delta}}= & g_{\alpha \overline{\bar{\beta}}} g_{\gamma \bar{\delta}}+g_{\alpha \bar{\delta}} g_{\bar{\beta} \gamma}-\frac{1}{K^{* 2}}\left(K^{*} K_{\alpha \bar{\beta} \gamma \bar{\delta}}^{*}-K_{\alpha \gamma}^{*} K_{\bar{\beta} \bar{\delta}}^{*}\right), \\
& +\sum \frac{1}{K^{* 4}} g^{\bar{\epsilon} \tau}\left(K^{*} K_{\alpha \gamma \bar{\epsilon}}^{*}-K_{\alpha \gamma}^{*} K_{\bar{\epsilon}}^{*}\right)\left(K^{*} K_{\bar{\beta} \bar{\delta} \tau}^{*}-K_{\bar{\beta} \bar{\delta}}^{*} K_{\tau}^{*}\right) .
\end{aligned}
$$

As in $\S 2$, we denote by $F$ the set of square integrable holomorphic $n$-forms on $M$. Let $z$ be any point of $M$ and let $F^{\prime}(z)$ be the set of elements of $F$ which vanish at $z$. Then $F^{\prime}(z)$ is a closed subspace of $F$ and the orthogonal complement to $F^{\prime}(z)$ is a complex 1-dimensional subspace of $F$. Let $h_{0}$ be a basis for this 1 -dimensional subspace with $\left(h_{0}, h_{0}\right)=1$. Then $h_{0}$ is unique up to a constant $c$ with $|c|=1$.

Let $F^{\prime \prime}(z)$ be the set of elements of $F^{\prime}(z)$ such that $d f^{*}=0$ at $z$, where $f^{*}$ is defined by $f=f^{*} d z^{1} \wedge \cdots \wedge d z^{n}$. This definition is independent of choice of coordinate system. The orthogonal complement to $F^{\prime \prime}(z)$ in $F^{\prime}(z)$ is a complex $n$-dimensional subspace. Let $h_{1}, \cdots, h_{n}$ be an orthonormal basis of this $n$-dimensional subspace. An easy calculation shows that (see §3)

$$
\sum g_{\alpha \bar{\beta}} d z^{\alpha} d \bar{z}_{\bar{\beta}}=\left(\sum_{k=1}^{n} d h_{k}^{*} d \bar{h}_{k}^{*}\right) / K^{*} \quad \text { at } z .
$$

Let $z^{1}, \cdots, z^{n}$ be a normal coordinate system at $z$; i.e., $g_{\alpha \bar{\beta}}=\delta_{\alpha \beta}$ (Kronecker delta) at $z$ in terms of this coordinate system. Then, applying a unitary transformation to the basis $h_{1}, \cdots, h_{n}$, we may assume that

$$
\partial h_{k}^{*} / \partial z^{j}=h_{0}^{*} \cdot \delta_{j k} \quad \text { at } z \quad(j, k=1, \cdots, n) .
$$

It follows, by a straightforward calculation, that

$$
R_{\alpha \bar{\beta} \gamma \bar{\delta}}=-\left(\sum_{j=n+1}^{\infty} h_{j \alpha \gamma}^{*} \bar{h}_{j \overline{\beta \delta}}^{*}\right) / K^{*}+\left(\delta_{\alpha \beta} \delta_{\gamma \delta}+\delta_{\alpha \delta} \delta_{\beta \gamma}\right)
$$

at $z$ with respect to a normal coordinate system at $z$, where 


$$
h_{j \alpha \gamma}^{*}=\partial^{2} h_{j}^{*} / \partial z^{\alpha} \partial z^{\gamma}, \quad \bar{h}_{j \bar{\beta} \delta}^{*}=\partial^{2} h_{j}^{*} / \partial \bar{z}^{\beta} \partial \bar{z}^{\delta} .
$$

Let $u=\sum_{\alpha=1}^{n} u^{\alpha}\left(\partial / \partial z^{\alpha}\right)_{z}$ be a holomorphic tangent vector at $z$ with unit length:

$$
\sum g_{\alpha \bar{\beta}} u^{\alpha} \bar{u}^{\beta}=1 \text {. }
$$

The holomorphic sectio..al curvature $S(u)$ with respect to $u$ is defined by

$$
S(u)=\sum R_{\alpha \bar{\beta} \gamma \bar{\delta}} u^{\alpha} \bar{u}^{\beta} u^{\gamma} \bar{u}^{\delta} .
$$

From the above expression of the Riemannian curvature form, it follows that

Theorem 4.4. Let $M$ be a complex manifold with (A.1 and 2). Then the holomorphic sectional curvature of $M$ is not greater than 2 and $(n+1) g_{\alpha \beta}-R_{\alpha \bar{\beta}}$ is a positive semidefinite hermitian form.

The second half of Theorem follows from the above expression of the Riemannian curvature and from $R_{\alpha \bar{\beta}}=\sum g^{\gamma \gamma \bar{\delta}} R_{\alpha \beta \gamma \bar{\delta}}$.

REMARK. For a bounded domain in $C^{n}$, the holomorphic sectional curvature is less than $2[5]$ and $(n+1) g_{\alpha \bar{\beta}}-R_{\alpha \bar{\beta}}$ is positive definite. It is very likely that most of domains have the negative sectional curvature.

5. Discontinuous groups. Let $d s^{2}$ be the Bergman metric of a complex manifold $M$ with (A.1 and 2). Let $D$ be a properly discontinuous group of holomorphic transformations of $M$, i.e.,

(i) If $z$ and $z^{\prime}$ are points of $M$ not congruent modulo $D$, then there are neighborhoods $U$ of $z$ and $U^{\prime}$ of $z^{\prime}$ such that $U \cap s U^{\prime}$ is empty for all $s$ in $D$.

(ii) For every $z_{0}$ in $M$, the isotropy group $D\left(z_{0}\right)$ of $D$ is finite and there exists a neighborhood $V$ of $z_{0}$, stable by $D\left(z_{0}\right)$, such that the relations

$$
s \in D, \quad z \in V \text { and } s(z) \in V
$$

imply $s \in D\left(z_{0}\right)$.

Since the group $G(M)$ of holomorphic transformations of $M$ is closed in the group of isometries of $M$, it can be shown that $D$ is properly discontinuous if and only if $D$ is discrete and closed in $G(M)$.

We shall mainly consider the case $M / D$ is compact. In this case, we shall call $D$ a uniform discontinuous group. If $M / D$ is compact, then $G(M) / D$ is compact; the converse is true if $G(M)$ is transitive on $M$.

The quotient space $M / D$ is a manifold if and only if no element of $D$ except the identity has fixed point in $M$. In general, $M / D$ is a so-called $V$ manifold [25]. If $M / D$ is compact, it is an algebraic variety imbedded in a projective space $[2 ; 12 ; 19]$.

THEOREM 5.1. If $D$ is a uniform discontinuous group of holomorphic transformations on a complex manifold $M$ with (A.1 and 2), then $M$ is a complete Kaehlerian space with respect to the Bergman metric. 
Proof. If $M / D$ is a manifold without singularities, then the theorem is obvious. However, the theorem is true in the general case. Let $z_{1}, z_{2}, \cdots$, $z_{k}, \cdots$ be a Cauchy sequence in $M$. Let $x_{1}, x_{2}, \cdots, x_{k}, \cdots$ be its image in $M / D$. Since $M / D$ is compact, the image sequence converges to a point $x_{0}$ in $M / D$. Let $y_{1}, y_{2}, \cdots$ be the set of points in $M$ which are projected into $x_{0}$. Let $N\left(y_{1}\right)$ be the $\epsilon$-neighborhood ( $\epsilon$ is a small positive number) of $y_{1}$ satisfying the condition (ii). Let $N\left(y_{k}\right)$ be the $\epsilon$-neighborhood of $y_{k}(k=2,3, \cdots)$. Then $N\left(y_{k}\right)$ satisfies also (ii). It can be easily shown that $N\left(y_{j}\right) \cap N\left(y_{k}\right)$ is empty if $j$ is different from $k$. Hence, there exist integers $j$ and $m_{0}$ such that, for $m>m_{0}, z_{m}$ is in $N\left(y_{j}\right)$. Since $N\left(y_{j}\right)$ is a finitely ramified covering of the $\epsilon$-neighborhood of $x_{0}$, the sequence $z_{1}, z_{2}, \cdots$ converges to $y_{j}$.

TheOREm 5.2. Under the same assumption as in Theorem 5.1, $\operatorname{det}\left(R_{\alpha \bar{\beta}}\right)$ is different from zero at some point of $M$.

Proof. We shall give a proof here under an additional assumption that $M / D$ is a manifold without singularities. In a general case, we need the method of Bailey [1]. Let $K$ be the kernel form of $M$ and let $v$ be the volume element of the Kaehlerian manifold $M$. Let $J$ be a positive function on $M$ defined by

$$
(-1)^{n^{2} / 2} K=J v \text {. }
$$

Since both $K$ and $v$ are invariant by $D$, so is $J$. Hence, $J$ is a well defined function on $M / D$. From

$$
\bar{\partial} \partial \log K^{*}=\bar{\partial} \partial \log v^{*}+\bar{\partial} \partial \log J,
$$

it follows that the cohomology class of $\rho=-(-1)^{1 / 2} \sum R_{\alpha \bar{\beta}} d z^{\alpha} \wedge d \bar{z}^{\beta}$ is equal to that of $\omega=(-1)^{1 / 2} \sum g_{\alpha \beta} d z^{\alpha} \wedge d \bar{z}^{\beta}$. Since $(\omega)^{n}$ is not cohomologous to zero, $(\rho)^{n}$ is not cohomologous to zero. (Here, $\omega$ and $\rho$ are considered as forms on $M / D$ rather than on $M$.) The latter is represented by a $2 n$-form

$$
\left(-(-1)^{1 / 2}\right)^{n^{2}} \operatorname{det}\left(R_{\alpha \bar{\beta}}\right) d z^{1} \wedge \cdots \wedge d z^{n} \wedge d \bar{z}^{1} \wedge \cdots \wedge d \bar{z}^{n} .
$$

This proves our theorem.

TheOREM 5.3. Under the same assumption as in Theorem 5.1, the largest connected group of isometries of $M$ is the same as the largest connected group of holomorphic transformations of $M$.

Proof. An immediate consequence of Theorem 5.2 and Theorem 4.2.

THEOREM 5.4. Under the same assumption as in Theorem 5.1, $M$ admits no Killing vector field (nor holomorphic vector field) commuting with $D$.

Proof. Note that if $M / D$ is a manifold, Theorem asserts the nonexistence of Killing vector field on $M / D$.

Evidently, every vector field on a compact manifold generates a 1- 
parameter group of global transformations. Therefore, any vector field on $M$ commuting with $D$ generates a 1-parameter group of global transformations of $M$, if $M / D$ is a compact manifold. An argument similar to the one used in the proof of Theorem 5.1 shows that this is true without the assumption that $M / D$ is a manifold with no singularities.

In order to simplify the language, we shall use the expression "a vector field on $M / D$ " in place of "a vector field on $M$ commuting with $D$." Since every holomorphic vector field on $M / D$ generates a 1-parameter group of global holomorphic transformations of $M$, it is a Killing vector field (i.e., infinitesimal isometries). We shall prove, therefore, only the nonexistence of Killing vector field on $M / D$.

Let $X$ be a Killing vector field on $M / D$. By Theorem 5.3, $X$ is a holomorphic vector field. Since $i X$ is also a holomorphic vector field on $M / D$, it generates a 1-parameter group of global holomorphic transformations of $M$. Therefore, $i X$ is a Killing vector field on $M$. Theorem 3.7 and Theorem 5.2 show that $X$ is a zero vector field.

Corollary. If $M / D$ is a compact manifold, then the group of isometries of $M / D$ is finite.

Proof. An element of $G(M)$ induces a transformation of $M / D$ if and only if it is in the normalizor $N(D)$ of $D$ in $G(M)$. Let $G^{0}(m)$ be the connected component of the identity of $G(M)$. Since $D$ is discrete in $G(M), N(D) \cap G^{0}(M)$ consists of those elements which commute with every element of $D$. Therefore, every Killing vector field on $M / D$ induces a Killing vector field on $M$ which commutes with $D$. By Theorem 5.4, there is no Killing vector field on $M / D$. The group of isometries of $M / D$ is therefore discrete; on the other hand, it is compact. Hence, it is finite.

THEOREM 5.5. Under the same assumption as in Theorem 5.1, the center of the group $G(M)$ of holomorphic transformations is discrete. More generally, the center of any subgroup of $G(M)$ which contains $D$ is discrete.

Proof. Let $G^{\prime}$ be a subgroup of $G(M)$ containing $D$. The center of $G^{\prime}$ commutes with $D$. Hence it is discrete by Theorem 5.4.

REMARK. Compare Theorem 5.5 with the following [20]:

Let $M$ be a complex manifold with (A.1 and 2) and assume $G(M)$ to be transitive on $M$. If $G^{\prime}$ is a subgroup of $G(M)$ transitive on $M$, then the center of $G^{\prime}$ is discrete. This fact can be proved as follows. Let $X$ be an infinitesimal transformation of $M$ generating a 1-parameter subgroup of the center of $G^{\prime}$. Since $i X$ commutes with every element of $G^{\prime}, i X$ generates a 1 -parameter group of holomorphic transformations of $M$. In fact, let $z_{0}$ be any, but fixed point of $M$ and suppose that $\exp (i t X) z_{0}$ is defined for $|t|<\epsilon$. Let $z$ be any point of $M$ and let $\phi$ be an element of $G^{\prime}$ such that $\phi\left(z_{0}\right)=z$. Then $\exp (i t X) z$ can be defined and is equal to $\phi\left(\exp (i t X) z_{0}\right)$ for $|t|<\epsilon$. Therefore, both $X$ 
and $i X$ are Killing vector fields. By Theorem 3.7 and Theorem 4.1, $X$ has to be a zero vector field.

ReMARK. If $M / D$ is compact, then $G(M) / D$ is compact; hence $G(M)$ is unimodular. (In general, a locally compact group admitting a uniform discrete subgroup is unimodular) $\left.{ }^{1}\right)$. If $G(M)$ is transitive and $M / D$ is compact, then $M$ is symmetric in the sense of E. Cartan by a theorem of Hano[14] .

We shall study the curvature again under the assumption that $M / D$ is compact. Let $J$ be a real valued function defined by (see $\$ 5$ )

$$
(-1)^{n^{2} / 2} K=J v \text {. }
$$

From

$$
g_{\alpha \bar{\beta}}=\frac{\partial^{2} \log J}{\partial z^{\alpha} \partial \bar{z}^{\beta}}-R_{\alpha \bar{\beta}}
$$

we obtain

$$
n=\Delta J-R,
$$

where $R=\sum g^{\alpha \bar{\beta}} R_{\alpha \bar{\beta}}$ is the scalar curvature. Since each term in the above equality is invariant by $D$, the equality holds on $M / D$. If $\Delta J \geqq 0$ on $M$ or $\leqq 0$ on $M$, then $J$ is constant by Theorem of E. Hopf [29]. Hence

THEOREM 5.6. Under the same assumption as in Theorem 5.1, if the scalar curvature $R$ satisfies

either $R \leqq-n$ everywhere on $M$,

or $R \geqq-n$ everywhere on $M$,

then $R_{\alpha \bar{\beta}}=-g_{\alpha \bar{\beta}}$, consequently $R=-n$.

REMARK. It can be also shown that the mean of $R$ is equal to $-n$ in the following sense:

$$
\int_{M / D} R \cdot v=-n \times(\text { volume of } M / D) \text {. }
$$

6. Domain of holomorphy and Bergman metric. Let $M$ be a bounded domain in $C^{n}$. Then the conditions (A.1 and 2) are obviously satisfied. Let $M^{*}$ be the envelope of holomorphy of $M$. Bremermann has proved [8] that the Bergman metric of $M$ can be extended to a Kaehlerian metric of $M^{*}$. Hence

TheOREM 6.1. If a bounded domain $M$ in $C^{n}$ is complete with respect to the Bergman metric, then it is a domain of holomorphy.

REMARK. Bremermann has shown also that the converse is not true.

(1) The author was informed of this fact by H. C. Wang. 
The following theorem of Siegel [27] is now an immediate consequence of Theorem 5.1 and Theorem 6.1.

Theorem 6.2. Let $M$ be a bounded domain in $C^{n}$. If there exists a proper discontinuous group $D$ of holomorphic transformations of $M$ such that $M / D$ is compact, then $M$ is a domain holomorphy.

Every homogeneous Riemannian space is complete. From Theorem 5.1 and Theorem 6.1, we obtain, therefore, the following

Theorem 6.3. If $M$ is a homogeneous bounded domain in $C^{n}$, then $M$ is a domain of holomorphy.

In the above three theorems, the assumption that $M$ is a "bounded domain in $C^{n}$ " can be replaced by the one that $M$ is a domain in a Stein manifold and satisfies (A.1 and 2). It is of interest to find the proof of these theorems which does not make use of the fact that $M$ is imbedded in a Stein manifold.

7. Infinite dimensional projective spaces. Let $H$ be a separable complex Hilbert space with orthonormal coordinate system $\zeta=\left(\zeta^{0}, \zeta^{1}, \zeta^{2}, \cdots\right)$. Let $\zeta$ and $\zeta^{\prime}$ be points in $H$ both different from zero. We say that they are equivalent if there exists a complex number $a$ such that $\zeta^{\prime}=a \zeta$. The quotient space of $H-\{0\}$ by this equivalence relation is the projective space $P(H)$. Let $S(H)$ be the unit sphere in $H$ with center at 0 . Then $P(H)$ is a quotient space of $S(H)$. The topology of $S(H)$ as a subspace of $H$ induces a topology on the quotient space $P(H)$. Then $S(H)$ is a principal fibre bundle over $P(H)$ with circle group.

Since $S(H)$ is a subspace of a metric space $H, S(H)$ itself is a metric space. The metric on $S(H)$ induces a metric on $P(H)$ as follows: if $x$ and $x^{\prime}$ are points in $P(H)$ (i.e., $x$ and $x^{\prime}$ are fibres in $\left.S(H)\right)$, distance $\left(x, x^{\prime}\right)=\inf _{\zeta \in x, \zeta^{\prime} \in x^{\prime}}$ distance $\left(\zeta, \zeta^{\prime}\right)$. As $H$ is complete and $S(H)$ is closed, $S(H)$ is a complete metric space. From the compactness of the fibre (=circle group) of $S(H)$, it follows that $P(H)$ is also complete.

We shall define another metric on $P(H)$ which is a generalization of the canonical Kaehler metric on finite dimensional projective space.

A continuous map $c$ of the real line $R$ into $H$ given by the equation $\zeta^{i}=\zeta^{i}(t)$ is said to be differentiable at $t=0$ if each $\zeta^{j}(t)$ is differentiable at $t=0$ and if $\sum\left|a^{j}\right|^{2}<\infty$, where $a^{j}=\left(d \zeta^{j} / d t\right)_{t=0}$. A continuous map $c$ of $R$ into $P(H)$ is said to be differentiable at $t=0$ if there exists a differentiable map $c^{\prime}$ of $R$ into $S(H)$ such that $p \circ c^{\prime}=c$, where $p$ is the projection of $S(H)$ onto $P(H)$.

Let $x$ be a point of $P(H)$ and let $C(x)$ be the set of continuous maps $c: R \rightarrow P(H)$ differentiable at $t=0$ with $c(0)=x$. Two elements $c_{1}$ and $c_{2}$ of $C(x)$ are, by definition, tangent to each other at $x$, if there exist $c_{1}^{\prime}$ and $c_{2}^{\prime}: R \rightarrow S(H)$ differentiable at $t=0$ with (i) $c_{1}^{\prime}(0)=c_{2}^{\prime}(0)$, (ii) $\left(d c_{1}^{\prime} / d t\right)_{t=0}$ 
$=\left(d c_{2}^{\prime} / d t\right)_{t=0}$, (iii) $p \circ c_{1}^{\prime}=c_{1}$ and $p \circ c_{2}^{\prime}=c_{2}$. We identify elements of $C(x)$ tangent to each other; each equivalence class is a tangent vector to $P(H)$ at $x$ and the tangent space to $P(H)$ at $x$ will be denoted by $T_{x}(P(H))$.

Let $x$ be a point of $P(H)$ and let $\zeta_{0}$ be a point of $S(H)$ representing $x$. The subspace of $H$ consisting of vectors orthogonal to $\zeta_{0}$ will be denoted by $T_{x}$ since it depends only on $x$. Then

$$
H=T_{x}+C \zeta_{0},
$$

where $C \zeta_{0}$ is the 1-dimensional subspace spanned by $\zeta_{0}$.

Let $\bar{x} \in T_{x}(P(H))$ and let $c: R \rightarrow S(H)$ with $c(0)=\zeta_{0}$ represent $\bar{x}$. If $c$ is given by $\zeta=\zeta(t)$, then $(d \zeta / d t)_{t=0}$ considered as an element of $H$, lies in $T_{x}$ and depends only on $\bar{x}$, as it can be easily verified. Thus, we obtain a mapping from $T_{x}(P(H))$ into $T_{x}$. It is not difficult to see that this mapping is a vector space isomorphism of $T_{x}(P(H))$ onto $T_{x}$.

Now, consider the quadratic differential form $d \sigma^{2}$ on $S(H)$ defined by

$$
d \sigma^{2}=\sum d \zeta^{i} d \bar{\zeta}^{i}-\left(\sum \bar{\zeta}^{i} d \zeta^{j}\right)\left(\sum \zeta^{k} d \bar{\zeta}^{k}\right) .
$$

As in the case of finite dimensional projective space, this quadratic differential form can be projected onto the base space $P(H)$. We shall denote by the same symbol $d \sigma^{2}$ the quadratic form on $P(H)$ obtained in this manner. From the Schwarz's inequality, it follows that $d \sigma^{2}$ is positive definite.

Let $c$ be a differentiable curve in $P(H)$, i.e., a differentiable map of a closed interval $[a, b]$ into $P(H)$. Then the length $L(c)$ of $c$ is defined as follows. Let $c^{\prime}$ be a curve in $S(H)$ such that $p \circ c^{\prime}=c$ and let $\zeta=\zeta(t)$ be the equation of $c^{\prime}$. Then

$$
L(c)=\int_{a}^{b}\left(\left(\sum \frac{d \zeta^{i}}{d t} \frac{d \bar{\zeta}^{i}}{d t}\right)-\left(\sum \bar{\zeta}^{j} \frac{d \zeta^{i}}{d t}\right)\left(\sum \zeta^{k} \frac{d \bar{\zeta}^{k}}{d t}\right)\right)^{1 / 2} d t .
$$

Let $x_{1}$ and $x_{2}$ be points of $P(H)$. The distance $d\left(x_{1}, x_{2}\right)$ between $x_{1}$ and $x_{2}$ is defined, as in the classical case, by

$$
d\left(x_{1}, x_{2}\right)=\inf L(c),
$$

where inf is taken over all differentiable curves joining $x_{1}$ and $x_{2}$. Then $P(H)$ is a metric space.

We shall see that uniform structure on $P(H)$ defined by the metric $d \sigma^{2}$ is the same as the one defined by the metric induced from that of $S(H)$. Let $x \in P(H)$ and $\zeta_{0} \in S(H)$ with $p\left(\zeta_{0}\right)=x$. By a proper choice of basis in $H$, we may assume that $\zeta_{0}=(1,0,0, \cdots)$. Then, on the tangent space at $x, d \sigma^{2}$ is reduced to

$$
d \zeta^{1} d \bar{\zeta}^{1}+d \zeta^{2} d \bar{\zeta}^{2}+\cdots
$$

This shows that the metric $d \sigma^{2}$ and the metric induced from that of $S(H)$ give the same distance for a pair of points infinitesimally near to each other. It 
follows that two metrics give rise to the same uniform structure on $P(H)$. Hence $P(H)$ is complete with respect to the generalized Kaehler metric $d \sigma^{2}$.

REMARK. Let $x_{1}$ and $x_{2}$ be any points of $P(H)$. Then, by a proper choice of basis in $H$, we may assume that points $(1,0,0, \cdots)$ and $\left(1 /\left(a^{2}+1\right)^{1 / 2}\right.$, $\left.a /\left(a^{2}+1\right)^{1 / 2}, 0, \cdots\right)$ in $S(H)$ represent $x_{1}$ and $x_{2}$ respectively, where $0 \leqq a$ $\leqq \infty$. The distance between $x_{1}$ and $x_{2}$ defined by two metrics is given by

$$
\begin{array}{cl}
\left(2-2 /\left(a^{2}+1\right)^{1 / 2}\right)^{1 / 2} & \text { (induced from the metric of } S(H) \text { ) } \\
\operatorname{Arctan} a & \text { (defined by } \left.d \sigma^{2}\right) .
\end{array}
$$

If we make the change of parameter by $a=\tan t$, then we obtain

$$
2 \sin t / 2 \text { and } t \text {. }
$$

This shows clearly that two metrics are almost identical in a small domain.

We summarize the results of this section in the following

Theorem 7.1. Let $H$ be a separable complex Hilbert space and let $P(H)$ be the projective space induced from $H$ (i.e., a point of $P(H)$ is a 1-dimensional subspace of $H)$. As $P(H)$ is a quotient space of the unit sphere $S(H)$ in $H$ such that $S(H)$ is a principal fibre bundle over $P(H)$ with circle group, $P(H)$ is complete with respect to the metric induced from that of $S(H),(S(H)$ being a subspace of a metric space $H$ ). The Kaehler metric $d \sigma^{2}$ on $P(H)$ (which is a natural generalization of the canonical Kaehler metric in a finite dimensional projective space) give the same uniform structure as the previously defined metric. In particular, $P(H)$ is complete with respect to the Kaehler metric $d \sigma^{2}$.

8. Imbedding of complex manifolds into $P(H)$. Let $M$ be a complex manifold and let $F$ be the Hilbert space of square integrable holomorphic $n$-forms in $M$. Let $H$ be the Hilbert space dual to $F$. Let $z^{1}, \cdots, z^{n}$ be a local coordinate in $M$ and let $z$ be a point of $M$ where this coordinate system is valid. Let $j^{\prime}$ be a mapping which sends $z$ into an element $j^{\prime}(z)$ of $H$ defined by

$$
\left\langle j^{\prime}(z), f\right\rangle=f^{*}(z) \quad \text { for } f=f^{*} d z^{1} \wedge \cdots \wedge d z^{n} \in F .
$$

This mapping $j^{\prime}$ depends on local coordinate. Evidently, $j^{\prime}(z)$ is different from zero for all $z$ if and only if $M$ satisfies (A.1). Assume that $M$ satisfies (A.1). Let $p^{\prime}$ be the natural projection of $H-\{0\}$ onto $P(H)$. It follows easily that $j=p^{\prime} \circ j^{\prime}$ is independent of local coordinate and is well defined all over $M$. The map $j: M \rightarrow P(H)$ is continuous and, moreover, complex analytic in an obvious sense. (The definition of complex analyticity of a mapping can be given in a similar way as the definition of differentiability given in $\$ 7$.

We define the the invariant quadratic differential form $d s^{2}$ on a complex manifold $M$ with (A.1). A simple calculation shows that 
THEOREM 8.1. The quadratic differential form $d s^{2}$ of $M$ with (A.1) is induced from the canonical Kaehler metric $d \sigma^{2}$ of $P(H)$ by $j ; d s^{2}=j^{*}\left(d \sigma^{2}\right)$.

In the same way as in the finite dimensional case, we can define the differential $\delta j$ of $j$ as a linear mapping of the tangent space to $M$ at $z$ into the tangent space to $P(H)$ at $j(z)$. Then, it is easy to see that

Theorem 8.2. The differential $\delta j$ of $j$ is nonsingular at every point of $M$ if and only if $M$ satisfies (A.2).

The above two theorems give us a geometric interpretation of the Bergman metric. If $M$ satisfies (A.1 and 2), then $j$ is an isometric immersion of $M$ into $P(H)$.

REMARK. Observe that $j$ preserves the distance between a point of $M$ and a point infinitesimally close to it; but the distance between two points $z$ and $z^{\prime}$ of $M$ is not less than the distance between $j(z)$ and $j\left(z^{\prime}\right)$, and not necessarily equal.

If $M$ satisfies (A.1 and 2), then $j$ is locally one-one in the sense that for every point of $M$, there is a neighborhood for it such that $j$ maps this neighborhood one-to-one into $P(H)$. It is, however, not necessarily injective in the large.

Let $z$ and $z^{\prime}$ be any points of $M$. Let $F^{\prime}(z)$ and $F^{\prime}\left(z^{\prime}\right)$ be the set of elements $f$ of $F$ which vanish respectively at $z$ and $z^{\prime}$. If $M$ satisfies (A.1), then $F^{\prime}(z)$ and $F^{\prime}\left(z^{\prime}\right)$ are subspaces of $F$ of codimension 1 . If $F^{\prime}(z)$ is contained in $F^{\prime}\left(z^{\prime}\right)$, then it is necessarily equal to $F^{\prime}\left(z^{\prime}\right)$; this implies that $j(z)$ is equal to $j\left(z^{\prime}\right)$. It follows that if $M$ satisfies (A.1) and $j$ is injective, then $M$ satisfies

(A.3). If $z$ and $z^{\prime}$ are two distinct points of $M$, then there is a square integrable holomorphic $n$-form $f$ such that $f(z) \neq 0$ and $f\left(z^{\prime}\right)=0$.

It is easy to see that, conversely, if $M$ satisfies (A.3), then $M$ satisfies automatically (A.1) and $j$ is injective. Therefore, if $M$ is a complex manifold satisfying (A.2 and 3), then $j$ is an isometrical imbedding of $M$ into $P(H)$.

9. A sufficient condition for $M$ to be complete. We have seen previously (§6) that if a bounded domain in $C^{n}$ is complete with respect to the Bergman metric, then it is a domain of holomorphy. But the converse is not true. In this section, we shall give a sufficient condition for a complex manifold $M$ with (A.1 and 2) to be complete with respect to the Bergman metric and shall prove that every bounded analytic polyhedron in $C^{n}$ is complete.

Let $M$ be a complex manifold satisfying (A.1 and 2) and $F$ the space of square integrable holomorphic $n$-forms on $M$. Let $K$ be the kernel form of $M$. If $f \in F$, we shall mean by $(f \wedge \bar{f}) / K$ a function $g$ on $M$ such that $f \wedge \bar{f}=g K$. Notice that

$$
0 \leqq(f \wedge \bar{f}) / K \leqq(f, f) .
$$

Theorem 9.1. A complex manifold $M$ with (A.1 and 2) is complete with respect to the Bergman metric if the following condition is satisfied. 
(A.4) $\left({ }^{2}\right)$. For every infinite sequence $S$ of points of $M$ which has no adherent point in $M$ and for each $f \in F$, there exists a subsequence $S^{\prime}$ of $S$ such that

$$
\lim _{S^{\prime}}(f \wedge \bar{f}) / K=0 .
$$

Proof. Suppose $M$ is not complete. Let $S$ be a Cauchy sequence in $M$ which has no limit point in $M$. Let $j$ be the natural map of $M$ into $P(H)$ defined in the preceding section. Since $S$ is a Cauchy sequence, $j(S)$ is also a Cauchy sequence in $P(H)$. From the completeness of $P(H)$ it follows that $j(S)$ has a limit point $x_{0}$ in $P(H)$. By a proper choice of basis in $H$, we may assume that $x_{0}$ is represented by a point $\zeta_{0}=(1,0,0, \cdots)$ of $H$. Take the dual basis $h_{0}, h_{1}, h_{2}, \cdots$ in $F$. Then

$$
\lim _{S}\left(h_{0} \wedge \bar{h}_{0}\right) / K=1 \text {. }
$$

Let $S^{\prime}$ be any subsequence of $S$. Then, $j\left(S^{\prime}\right)$ and $j(S)$ have the same limit point in $P(H)$. Hence

$$
\lim _{S^{\prime}}\left(h_{0} \wedge \bar{h}_{0}\right) / K=1 .
$$

This completes the proof of our theorem.

Let $D$ be a domain in $C^{n}$ and let $f_{q}, q=1, \cdots, k$, be $k$ holomorphic functions on $D$. Let $M$ be a connected component of the region $\bigcap_{q}\left\{z \in D ;\left|f_{q}(z)\right|\right.$ $<1\}$ and assume that the closure of $M$ in $C^{n}$ is compact and is contained in $D$. Then, we call $M$ a bounded analytic polyhedron.

THEOREM 9.2. Every bounded analytic polyhedron in $C^{n}$ satisfies (A.4); hence it is complete with respect to the Bergman metric.

We shall prove first the following

Lemma. Let $M$ be a bounded analytic polyhedron in $C^{n}$. Then, for every infinite sequence $S$ of points of $M$ which has no adherent point in $M$, there exists a subsequence $S^{\prime}=\left\{z_{1}, z_{2}, \cdots\right\}$ of $S$ with the following property: given a positive number $\epsilon$ and an element $g$ of $F$, there exist an integer $N$ and an element $f$ of $F$ with $\|f\|=1$ such that $\left|(g / f)\left(z_{j}\right)\right|<\epsilon$ for $j>N$.

Proof. Notice first that $g / f$ denotes a meromorphic function on $M$ defined by $(g / f) f=g$. Since the closure of $M$ is compact, $S$ has a subsequence $S^{\prime}$ which converges to a point $z_{0}$ of the boundary of $M$. Then $\left|f_{q}\left(z_{0}\right)\right|=1$ for some $q$, say $q=1$ for the sake of simplicity. Let $M_{a}=\left\{z \in M ;\left|f_{1}(z)\right|<a\right\}$ for $0<a<1$. Since $M_{a} \rightarrow M$ as $a \rightarrow 1$, there exists an $a$ such that

$$
\left|\int_{M-M_{a}} g \wedge \bar{g}\right|<\epsilon^{2} / 8
$$

Let $m$ be a positive integer such that $\left\|a^{m} g\right\|^{2} \leqq \epsilon^{2} / 8$. Then

${ }^{2}$ Roughly speaking, the condition (A.4) means that the isometric imbedding of $M$ into $P(H)$ is closed. 


$$
\begin{aligned}
\left\|\left(f_{1}\right)^{m} g\right\|^{2} & \leqq \int_{M-M_{a}}\left((-1)^{1 / 2}\right)^{n^{2}} g \wedge \bar{g}+\int_{M_{a}}\left((-1)^{1 / 2}\right)^{n^{2}} a^{2 m} g \wedge \bar{g} \\
& <\epsilon^{2} / 8+\epsilon^{2} / 8=\epsilon^{2} / 4 .
\end{aligned}
$$

Let $c$ be a positive constant such that $\left\|c\left(f_{1}\right)^{m} g\right\|=1$. Then $c \epsilon \geqq 2$. Let $f=c\left(f_{1}\right)^{m} g$. Then $|g / f|=\left|1 /\left(c f_{1}^{m}\right)\right|<\left|\epsilon /\left(2 f_{1}^{m}\right)\right|$. Let $N$ be an integer such that $f_{1}^{m}\left(z_{j}\right)>1 / 2$ for $j>N$. This completes the proof of our lemma.

Now, we shall show that $M$ satisfies (A.4). Let $S^{\prime}$ be a subsequence of $S$ which converges to a boundary point $z_{0}$ of $M$. Let $g$ be any element of $F$. For any positive number $\epsilon$, there exist an element $f$ of $F$ and an integer $N$ satisfying the assertion of the lemma. Take an orthonormal basis $h_{0}, h_{1}, h_{2}, \cdots$ of $F$ such that $h_{0}=f$. Then, it is obvious that $(g \wedge \bar{g}) / K \leqq(g \wedge \bar{g}) /(f \wedge \bar{f})$. Hence

$$
\left(g\left(z_{j}\right) \wedge \bar{g}\left(\bar{z}_{j}\right)\right) / K\left(z_{j}, \bar{z}_{j}\right) \leqq \epsilon^{2} \quad \text { for } j>N .
$$

This proves that $M$ satisfies (A.4).

Now, we know that

(i) Every bounded analytic polyhedron in $C^{n}$ satisfies (A.4).

(ii) Every bounded domain in $C^{n}$ satisfying (A.4) is complete with respect to the Bergman metric.

(iii) Every bounded domain in $C^{n}$ which is complete with respect to the Bergman metric, is a domain of holomorphy.

(iv) Every domain of holomorphy $M$ in $C^{n}$ can be approximated by a sequence of analytic polyhedron $M_{j}$ in such a way that

$$
\bar{M}_{j} \subset M_{j+1}, \quad M_{j} \subset M \text { and } M=\lim M_{j} .
$$

The converse of (iii) is not true as stated in $\S 6$. The converse of (i) is not true (see Theorem 9.3). I do not know whether the converse of (ii) is true or not; it is very likely that the converse of (ii) holds.

It should be remarked [13] that every domain of holomorphy is complete with respect to some Kaehler metric.

The method of proof of the lemma is very similar to that used by Bremermann [8] who has shown that the kernel function goes to the infinity at every boundary point of an analytic polyhedron. As we have remarked before, the kernel function is not intrinsically defined. Moreover, the above theorem implies Bremermann's result: i.e., let $f$ be $d z^{1} \wedge \cdots \wedge d z^{n}$ in (A.4), where $z^{1}, \cdots, z^{n}$ is the Euclidean coordinate system in $C^{n}$.

We shall generalize Theorem 9.2 as follows.

Let $D$ be a domain in $C^{n}$ and let $f_{q},(q=1, \cdots, k)$, be a real analytic function on $D$ which can be written in the following form:

$$
f_{q}=\sum_{j=1}^{\infty} f_{q j} \bar{f}_{q j}
$$

where each $f_{q j}$ is holomorphic on $D$ and the sum is convergent uniformly on 
every compact set in $D$. Let $M$ be a connected component of the set

$$
\left\{z \in D ;\left|f_{q}(z)\right|<1 \text { for } q=1, \cdots, k\right\}
$$

and assume that the closure of $M$ in $C^{n}$ is compact and is contained in $D$. Then, we shall call $M$ a generalized analytic (bounded) polyhedron. It is clear that every analytic polyhedron is a generalized analytic polyhedron.

THEOREM 9.3. Every bounded generalized analytic polyhedron in $C^{n}$ satisfies (A.4); hence it is complete with respect to the Bergman metric.

Proof. Given an infinite sequence $S$ described in (A.4), let $S^{\prime}$ be a subsequence which converges to a boundary point $z_{0}$ of $M$. Then $\left|f_{q}\left(z_{0}\right)\right|=1$ for some $q$, say $q=1$. Consider a unitary transformation:

$$
\begin{aligned}
& f_{1}^{\prime}=\sum a_{1 j} f_{1 j}, \\
& f_{2}^{\prime}=\sum a_{2 j} f_{1 j},
\end{aligned}
$$

where $\left(a_{i j}\right)$ is an (infinite) unitary matrix. By a proper choice of $\left(a_{i j}\right)$, we may assume that $f_{1}^{\prime}\left(z_{0}\right)=1$ and $f_{2}^{\prime}\left(z_{0}\right)=f_{3}^{\prime}\left(z_{0}\right)=\cdots 0$. In the proof of the preceding lemma, we replace $f_{1}$ by $f_{1}^{\prime}$. Then the same argument is valid and proves our theorem.

We conjecture that the completeness with respect to the Bergman metric implies (A.4). In order to make this conjecture plausible, we shall prove the following

THEOREM 9.4. Let $M$ be a complex manifold with (A.1 and 2). If the group of holomorphic transformations is transitive on $M$, then $M$ satisfies (A.4).

Proof. Let $S$ be an infinite sequence of points of $M$ which has no adherent point in $M$. Given a positive number $\epsilon$, we can choose a subsequence $S^{\prime}$ $=\left\{z_{1}, z_{2}, \cdots\right\}$ of $S$ such that $N\left(z_{i}\right) \cap N\left(z_{j}\right)$ is empty for $i \neq j$, where $N\left(z_{j}\right)$ is the $\epsilon$-neighborhood of $z_{j}$ with respect to the Bergman metric. (Here, we make use of the completeness of $M$.) Let $z^{1}, \cdots, z^{n}$ be a coordinate system valid in $N\left(z_{1}\right)$. Let $\phi_{j}$ be a holomorphic transformation of $M$ such that $\phi_{j}\left(z_{j}\right)=z_{1}$, $(j=2,3, \cdots)$. Then $\phi_{j}\left(N\left(z_{j}\right)\right)=N\left(z_{1}\right)$ and $z^{1}, \cdots, z^{n}$ can be considered also as a coordinate system in $N\left(z_{j}\right)$. (We write $z^{1}, \cdots, z^{n}$ instead of $\phi_{j}^{*}\left(z^{1}\right), \cdots$, $\left.\phi_{j}^{*}\left(z^{n}\right)\right)$. Put

$$
K=K_{j}^{*} d z^{1} \wedge \cdots \wedge d \bar{z}^{n} \quad \text { in } N\left(z_{j}\right) .
$$

Since $K$ is invariant by $\phi_{j}$ and the coordinate system $z^{1}, \cdots, z^{n}$ in $N\left(z_{1}\right)$ is mapped into the coordinate system $z^{1}, \cdots, z^{n}$ in $N\left(z_{n}\right)$ by $\phi_{j}$, we have that $K_{1}^{*}=\phi_{j}^{*}\left(K_{j}^{*}\right)$. In particular,

$$
K_{1}^{*}\left(z_{1}, \bar{z}_{1}\right)=K_{2}^{*}\left(z_{2}, \bar{z}_{2}\right)=\cdots .
$$

Let $f$ be an element of $F$. Put 


$$
a_{j}=\left((-1)^{1 / 2}\right)^{n^{2}} \int_{N\left(z_{j}\right)} f \wedge \bar{f}
$$

Since $f$ is square integrable, $a_{j} \rightarrow 0$ as $j \rightarrow \infty$. Put

$$
f=f_{j}^{*} d z^{1} \wedge \cdots \wedge d z^{n} \quad \text { in } N\left(z_{j}\right) .
$$

Then (see p. 117 of [6])

$$
\left|f_{j}^{*}\left(z_{j}\right)\right|^{2} \leqq c a_{j},
$$

where $c$ is a constant independent of $j$. Hence $f_{j}^{*}\left(z_{j}\right) \rightarrow 0$ as $j \rightarrow \infty$. This proves our theorem.

THEOREM 9.5. Let $M$ be a complex manifold with (A.1 and 2). If $M$ admits a proper discontinuous group $D$ of holomorphic transformations such that $M / D$ is compact, then $M$ satisfies (A.4).

Proof. For the sake of simplicity, we shall assume that $M / D$ has no singular point. In the general case, we use the argument in the proof of Theorem 5.1. Let $S$ be an infinite sequence of points of $M$ which has no adherent point. Let $p$ be the projection of $M$ onto $M / D$. We can choose a subsequence $S^{\prime}=\left\{z_{1}, z_{2}, \cdots\right\}$ of $S$ such that $p\left(z_{1}\right), p\left(z_{2}\right), \cdots$ converges to a point $x_{0}$. Let $z^{1}, \cdots, z^{n}$ be a coordinate system in a neighborhood $N\left(x_{0}\right)$ of $x_{0}$. Choosing $S^{\prime}$ and $N\left(x_{0}\right)$ properly, we can find a neighborhood $N\left(z_{j}\right)$ of $z_{j}$ in such a way that $p$ maps $N\left(z_{j}\right)$ homeomorphically onto $N\left(x_{0}\right)$ and that $N\left(z_{i}\right) \cap N\left(z_{j}\right)$ is empty for $i \neq j$. We shall denote also by $z^{1}, \cdots, z^{n}$ the coordinate system in $N\left(z_{j}\right)$ induced from the system $z^{1}, \cdots, z^{n}$ in $N\left(x_{0}\right)$ by $p$. Put

$$
K=K_{j}^{*} d z^{1} \wedge \cdots d z^{n} \wedge d \bar{z}^{1} \wedge \cdots \wedge d \bar{z}^{n} \quad \text { in } N\left(z_{j}\right) .
$$

If $K_{1}^{*} \geqq a>0$ on $N\left(z_{1}\right)$, then $K_{j}^{*} \geqq a>0$ on $N\left(z_{j}\right)$ as $K$ is invariant by $D$. By a reasoning similar to that in Theorem 9.4, we can show that, for any $f \in F$, $f_{j}^{*}\left(z_{j}\right) \rightarrow 0$ as $j \rightarrow \infty$, where $f_{j}^{*}\left(z_{j}\right)$ is defined in the same way as in Theorem 9.4. This completes the proof.

\section{Examples.}

(A). Bounded domains in a Stein manifold. Let $V$ be an $n$-dimensional Stein manifold and $K(V)$ the canonical complex line bundle over $V$, i.e., the bundle of holomorphic $n$-forms on $V$. As assured by Theorem A of Cartan-Serre, $K(V)$ admits a sufficiently many holomorphic cross-sections, i.e., holomorphic $n$-forms on $V$. They are, however, not square integrable in general. Let $M$ be a bounded domain in $V$. Then, every holomorphic $n$-form on $V$ is square integrable on $M$. It can be easily shown that

TheORem 10.1. Every bounded domain in a Stein manifold satisfies (A.1 and 2).

(B). Nonsingular hypersurfaces of Degree $>n+2$ in $P_{n+1}(C)$.

THeOREM 10.2. Let $M$ be a nonsingular hypersurface of degree $>n+2$ in the 
$(n+1)$-dimensional complex projective space $P_{n+1}(C)$. Then $M$ satisfies (A.1, 2 and 3).

We shall only indicate the outline of the proof without going into the detail. We denote $P_{n+1}(C)$ by $P_{n+1}$. Then, $C^{n+2}-\{0\}$ can be considered, in a natural way, as a principal fibre bundle over $P_{n+1}$ with group $C^{*}$ (the multiplicative group of nonzero complex numbers). Let $E$ be the complex line bundle associated with this principal fibre bundle. Let $K\left(P_{n+1}\right)$ be the canonical complex line bundle over $P_{n+1}$. Then, by an easy calculation,

$$
K\left(P_{n+1}\right)=-(n+2) E .
$$

(The set of complex line bundles over a complex manifold forms an abelian group. The above notation should be understood in this sense [18].) Let $r$ be the degree of $M$. Then the line bundle given by the divisor $M$, denoted by $[M]$, is equal to $r E$. Let $K(M)$ be the canonical line bundle over $M$. Then

$$
K\left(P_{n+1}\right)=K(M)-[M] \quad \text { on } M \text {. }
$$

Hence

$$
K(M)=(r-n-2) E \mid M .
$$

From this relation it follows that there is a natural one-to-one correspondence between the set of holomorphic $n$-forms and the set of polynomials of degree $(r-n-2)$ in $(n+2)$-variables, provided that $(r-n-2)>0$.

Let $H$ be the set of polynomial function $f$ defined on $C^{n+2}$. Every point $x$ of $C^{n+2}$ can be considered as an element of the dual space of $H$ as follows:

$$
\langle x, f\rangle=f(x) .
$$

This mapping (injective) from $C^{n+2}$ into $H^{*}$ (the dual to $H$ ) induces a mapping $j^{*}$ of $P_{n+1}(C)$ into $P_{N}(C)$, where $N+1$ is the dimension of $H$. Evidently, $j^{*}$ is injective. Let $j^{\prime}$ be the injection map of $M$ into $P_{n+1}(C)$. Then, the natural imbedding $j$ of $M$ into $P_{N}(C)$ defined in $\S 8$ is the composed map $j^{*} \circ j^{\prime}$. If $r=n+3$, then $j=j^{\prime}$.

(C). Examples of Stein manifolds which are not complete with RESPECT To THE Bergman metric. Let $M$ be a bounded domain in the complex plane $C$. It is always a domain of holomorphy. If $M$ is complete with respect to the Bergman metric, then let $M^{\prime}$ be $M-a_{1}, \cdots, a_{k}$, where $a_{1}, \cdots, a_{k}$ are points of $M$. By Theorem $3.3, M^{\prime}$ is not complete. The example of Bremermann [8] is a typical one of this category.

More generally, let $M$ be a domain in $C^{n}$ which is complete with respect to the Bergman metric. It is a domain of holomorphy. Let $M^{\prime}$ be the zeros of a holomorphic function $f$ defined on $M$. Then, $M-M^{\prime}$ is a domain of holomorphy. Since $M^{\prime}$ is a subvariety of $M$, the Bergman metric of $M-M^{\prime}$ is the restriction of that of $M$ by Theorem 3.3. Hence $M-M^{\prime}$ is not complete.

Let $M$ be a nonsingular hypersurface of degree $>n+2$ in $P_{n+1}(C)$. Since 
$M$ is compact, $M$ is complete with respect to the Bergman metric. Let $M^{\prime}$ be $M-M \cap S$, where $S$ is a hyperplane in $P_{n+1}(C)$; i.e., $M^{\prime}$ is an affine algebraic manifold. Then $M^{\prime}$ is a Stein manifold as it can be easily seen. Again, by Theorem $3.3, M^{\prime}$ is not complete.

\section{BIBLIOGRAPHY}

1. W. L. Baily, Jr., The decomposition theorem for V-manifolds, Amer. J. Math. vol. 78 (1956) pp. 862-888. 430.

2. - On the imbedding of V-manifolds in projective space, ibid. vol. 79 (1957) pp. $403-$

3. S. Bergman, Ueber die Kernfunktion eines Bereiches und ihr Verhalten am Rande, J. Reine Angew. Math. vol. 169 (1933) pp. 1-42; vol. 172 (1935) pp. 89-128.

4. - Sur les fonctions orthogonales de plusieurs variables complexes, Mem. Sci. Math. Paris, no. 106, 1947.

5. — - Sur la fonction-noyau d'un domaine . . . , ibid, no. 108, 1948.

6. S. Bochner and W. T. Martin, Several complex variables, Princeton, 1948.

7. A. Borel, Les fonctions automorphes de plusieurs variables complexes, Bull. Soc. Math. France vol. 80 (1952) pp. 167-182.

8. H. J. Bremermann, Holomorphic continuation of the kernel function and the Bergman metric in several complex variables, Lectures on Functions of a Complex Variable, Michigan, 1955, pp. 349-383.

9. E. Cartan, Sur les domaines bornés homogènes de l'espace de n variables complexes, Abh. Math. Sem. Univ. Hamburg vol. 11 (1935) pp. 116-162.

10. H. Cartan, Sur les groupes de transformations analytiques, Actualités Sci. Ind., no. 198, 1935.

11. - Variétés analytiques complexes et cohomologie, Colloque sur les Fonctions de Plusieurs Variables, Bruxelles, 1953.

12. - Quotient d'un espace analytique par un groupe d'automorphismes, Alg. Geometry and Topology (Symposium in honor of S. Lefschetz), Princeton, 1957.

13. H. Grauert, Charakterisierung der Holomorphiegebiete durch die vollstaendige Kaehlersche Metrik, Math. Ann. vol. 131 (1956) pp. 38-75.

14. J. Hano, On Kaehlerian homogeneous spaces of unimodular Lie groups, Amer. J. Math. vol. 79 (1957) pp. 885-900.

15. S. Kobayashi, Espaces affines et riemanniennes symétriques, Nagoya Math. J. vol. 9 (1955) pp. 25-37.

16. - The theory of connections, Ann. Mat. Pura Appl. vol. 43 (1957) pp. 119-194.

17. S. Kobayashi and K. Nomizu, On automorphisms of a Kaehlerian structure, Nagoya Math. J. vol. 11 (1957) pp. 115-124.

18. K. Kodaira, and D. C. Spencer, Group of complex line bundles over compact Kaehler varieties, Proc. Nat. Acad. Sci. U.S.A. vol. 39 (1953) pp. 868-872. $28-48$.

19. K. Kodaira, On Kaehler varieties of restricted type, Ann. of Math. vol. 60 (1954) pp.

20. J. L. Koszul, Sur la forme hermitienne canonique des espaces homogènes complexes, Canad. J. Math. vol. 7 (1955) pp. 562-576.

21. A. Lichnerowicz, Sur les groupes d'automorphismes de certaines variêtés kaehleriennes, C.R. Acad. Sci. Paris vol. 239 (1954) pp. 1344-1345.

22. J. Mehring and F. Sommer, Kernfunktion und Hullenbildung in der Funktionentheorie mehrerer Veraenderlichen, Math. Ann. vol. 131 (1956) pp. 1-16.

23. S. Myers, and N. Steenrod, The group of isometries of a Riemannian manifold, Ann. of Math. vol. 40 (1939) pp. 400-416. 
24. K. Morita, On the kernel functions of symmetric domains, Sci. Rep. Tokyo Kyoiku Daigaku Sect. A. vol. 5 (1956) pp. 190-212.

25. I. Satake, On a generalization of the notion of manifold, Proc. Nat. Acad. Sci. U.S.A. vol. 42 (1956) pp. 359-363.

26. J. A. Schouten, and K. Yano, On pseudo Kaehlerian spaces admitting a continuous group of motions, Indag. Math. vol. 17 (1955) pp. 565-570.

27. C. L. Siegel, Analytic functions of several complex variables, Institute for Advanced Study, Princeton, 1948-1949.

28. G. A. Washnitzer, Dirichlet principle for analytic functions of several complex variables, Ann. of Math. vol. 61 (1955) pp. 190-195.

29. K. Yano and S. Bochner, Curvature and Betti numbers, Annals of Mathematics Studies, no. 32 .

Institute for Advanced Study,

Princeton, N. J. 